\title{
Assessing inflammation in obese patients using DII dietary inflammatory index - A pilot cross sectional study in Jeddah, Saudi Arabia in the year 2017
}

\author{
Noura M S Eid ${ }^{1, *}$, Al-Rotana Albadri ${ }^{1}$, Kholoud Alshobragi ${ }^{1}$, Nadin Sharqawi ${ }^{1}$, Razan Albar ${ }^{1}$, Abdulmalik Altaf ${ }^{2}$ \\ ${ }^{1}$ Faculty of Applied Medical Sciences, Department of Clinical Nutrition, King Abdulaziz University, Jeddah, Saudi Arabia \\ ${ }^{2}$ Faculty of Medicine, Department of Surgery, King Abdulaziz University, Jeddah, Saudi Arabia \\ *Corresponding author: ooaeid2@kau.edu.sa
}

\begin{abstract}
Obesity is associated with chronic low-grade inflammation caused by progressive infiltration in adipose tissue macrophage (increase M1 pro-inflammatory and decrease M2 anti-inflammatory). In this study, we aim to assess the inflammatory status of obese patients and the impact of using resveratrol as a therapeutic approach. A cross sectional trial on 10 patients from surgical word in KAUH were assessed by DII and blood test to measure there inflammatory status. In addition, a questionnaire is used to observe their social habits and medical history. Then an ex vivo study will be carried to assess the effect of resveratrol of adipose tissue macrophage. Results have shown that all volunteers had pro- inflammatory score of DII and high CRP. There was no association between types and amount of polyphenols intake and level of CRP, except flavonols was strongly associated negatively with CRP $\left(\mathrm{p}<0.05, \mathrm{R}^{2}=0.425\right)$. DII can be used in the future as a new tool in the routine assessment of the inflammatory potential for the diet, which can be applied to obese population in Saudi Arabia. In the next step of this research, we will study the therapeutic application of resveratrol to treat obesity from inflammatory aspect.
\end{abstract}

Keywords: obesity, inflammation, CRP, diet inflammatory index, polyphenols

Cite This Article: Noura M S Eid, Al-Rotana Albadri, Kholoud Alshobragi, Nadin Sharqawi, Razan Albar, and Abdulmalik Altaf, "Assessing inflammation in obese patients using DII dietary inflammatory index - A pilot cross sectional study in Jeddah, Saudi Arabia in the year 2017." Journal of Food and Nutrition Research, vol. 6, no. 3 (2018): 137-145. doi: 10.12691/jfnr-6-3-1.

\section{Introduction}

\subsection{Obesity}

Obesity is considered as one of the major public health problems worldwide. According to the World Health Organization, Several epidemiological studies showed that obesity has doubled between 1980 and 2014 [1]. Where the prevalence in the Saudi population aged 15 years or older is $28.7 \%$ Or 3.6 million [2]. Obesity is considered as a leading cause of chronic diseases such as high risk of coronary heart disease, certain types of cancers, shorter life expectancy, type 2 diabetes and insulin resistance [3]. The global surge in obesity can be attributed largely to the increased consumption of high fat diets in combination with sedentary lifestyles [3]. The development of obesity is related to the passive role of fat, stored in adiposities, where the metabolism changes during the enlargement of adipose tissue process associated to obesity [4]. In recent years, several hypotheses have been offered to explain obesity-related chronic low-grade inflammation especially among white adipose tissue illustrating a strong relation between metabolism and immunity [5]. However, the mechanism of obesity related chronic inflammation refers to the progressive infiltration of immune cells particularly macrophage, it increases within obese adipose tissue emerged as a result of a disproportion between proand anti-inflammatory cytokines production leading to dysfunction and systemic inflammation [3]. Obesity is defined as excessive fat accumulation in the body that impairs health [6]. The normal levels of body fat in young adult is considered being $12-20 \%$ of body weight in males and $20-30 \%$ in females, while levels in obese males $>25 \%$ of body weight and $>33 \%$ in females [7]. One of the major causes of obesity is an energy imbalance between the expended and consumed calories. Globally the intake of energy dense foods that are high in fat coupled with physical inactivity has increased due to the sedentary nature of work forms, altering the transportation modes and increasing urbanization. Obese people compared to those with healthy weights are at a higher risk of developing several serious diseases and health problem such as cardiovascular diseases, diabetes, musculoskeletal disorders and some forms of cancers. Obesity can be prevented through supportive environments and communities which can shape people's choices by making healthy food selection available and engaging the public in regular physical activity. At the individual level people can follow general recommendations such as increasing fruits and vegetables consumption, limiting energy intake from sugars and fats and increasing physical activity (150 minutes per week for adults). It is important at the societal 
level to support individuals in following the above recommendation through political collaboration and increased commitment of many private and public stakeholders. Ensure that physical activity and healthy food choices are affordable, available and accessible at all society levels [6].

\subsection{Inflammation}

Inflammation is the main mechanism related to pathogen killing, tissue repair, and maintaining the homeostasis mechanisms of infected or damaged tissue. In addition, it produces a number of chemical mediators that cause self-regulation of inflammatory response by secreting anti-inflammatory cytokines and preventing proinflammatory signaling. However, inflammation may be associated with pathological effects in low-grade inflammation [8]. It is associated with many chronic issues such as metabolic syndrome, and type 2 diabetes [9]. Such low-grade inflammation is characterized by adipose tissue macrophage (ATM) infiltrations [10]. Specifically, white adipose tissue (WAT) produces a number of factors called adipokines that play a significant role in obesity associated with insulin resistance and/or cardiovascular disease. Consequently, the level of some pro-inflammatory cytokines are increased, such as tumor necrosis factor $\alpha$

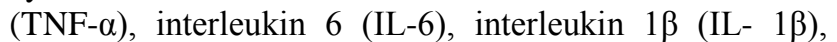
C-reactive protein (CRP), and other inflammatory markers $[11,12]$. Recent research has provided that adipose tissue inflammation is the main mechanism that cause complications during obesity such as type 2 diabetes [13,14], which is associated with pro-inflammatory action of bone marrow derived white adipose tissue macrophage (ATM) [15]. Inflammation occurs firstly due to the initiation of macrophage recruitment by expansion of adipose tissue due to hypertrophy and hyperplasia of adipocytes, which is caused by fatty acid flux, vascularization, increased leptin secretion, hypoxia and adipocytes cell death. The death of adipocytes leads to the production of a crown-like structure (CLS) around the dead cell and it is mostly consisting of macrophages. The visceral and epididymis fat depots have more adipose tissue death, inflammatory cytokines and accumulation of (ATM) than subcutaneous adipose tissue. Secondly, ATM themselves cause propagation of signals that lead to producing new macrophages at the same time, and then adipocytes initiate macrophages recruitment. To support this mechanism, recent study on lethal yellow (AY) mouse shows that there is a strong correlation between ATM content and adipose tissue mass when the total fat ranges from 2 to 15 g [16]. In addition, studies have shown that adipose tissue remodeling has a strong association with decreasing the number of ATM during obesity [17]. The mechanism of remodeling is identified by the macrophage phenotype in adipose tissue, which includes M1 and M2. M1 macrophage, which is a Pro-inflammatory or "classically activated" macrophage, is induced by lipopolysaccharides (LPS) and interferon- $\gamma$ (IFN- $\gamma$ ), and stimulates the production of tumor necrosis factor $\alpha(\mathrm{TNF}-\alpha)$ and interleukin 6 (IL-6) those may be associated with the initiation of insulin resistance and production of reactive oxygen species like nitric oxide (NO) due to stimulation of iNOS (Nos2). Whereas M2, exhibits an opposite effect, which is the Anti-inflammatory or "alternatively activated" macrophage that is generated by exposure to interleukin 4 (IL-4) and interleukin 13 (IL-13). In addition, it is stimulating the production of interleukin 10 (IL-10) and interleukin 1 (IL-1) receptors those may be associated with repairing and remodeling of the tissue. Also, M2 produces arginase enzymes that block the activity of iNOS via many mechanisms [10,18,19]. A study carried out by saltiel and colleagues showed that lean mice ATMs secrete high levels of high arginase-1 and IL-10, while the high-fat feed mice express low levels of arginase- 1 and IL-10 and high levels of TNF- $\alpha$ and inducible nitric oxide synthase (iNOS) [10]. Researchers suggested an imbalance between $\mathrm{M} 1$ and $\mathrm{M} 2$ to be associated with such inflammatory status [20]. Such trials prove that M1 macrophages are most likely to cause inflammation and insulin resistance in adipose tissue, while M2 macrophages are associated with preventing the increase in adipose tissue size. Thus, the phenotype of macrophages (M1 and M2) will indicate to control inflammation in adipose tissue and may associate with tissue repair and remodeling that occur during adipose tissue hypertrophy [21]. In vitro studies show that using luteolin (food-derived flavonoid) on 3T3-L1 adipocytes and RAW264 macrophages stop the activation of proinflammatory markers like TNF- $\alpha, \mathrm{MCP}-1$, and nitric oxide (NO) in adipose tissue macrophage [22]. Another study has investigated the effect obese mice which shows that capsaicin (a spicy component of hot peppers) reduces the level of MCP-1, IL-6, and COX-2 gene in adipose tissue. Also, it significantly decreases macrophage infiltration into adipose tissue [23]. Shirakawi et al study on high fat feed rodents show that monounsaturated fatty acid (MUFA) will prevent excessive adipocytes and prevent the infiltration of M1 macrophage and CD8+ T cell [24]. Little studies have been investigated using different type of polyphenols showing significant effect on adipose tissue macrophage infiltration. A study using grape powder extract (GPE) will decrease the expression of inflammatory markers such as IL-6, IL-8, IL-1 $\beta$, and TNF- $\alpha$ in adipose tissue macrophage [25]. In vitro studies show that using cocoa polyphenols extract on 3T3-L1 preadipocytes will inhibit the adipogenesis process. This result is supported by in vivo studies using the cocoa polyphenols extract on high fat fed mice shows inhibition in the weight gain [26].

\subsection{Dietary Inflammatory Index (DII)}

Dietary inflammatory index is a tool that measures inflammatory potential in an individual's diet quality from maximally anti-inflammatory (negative or healthier DII scores) to maximally pro- inflammatory (positive or unhealthy DII scores) for 45 food items and it predicts the level of six specific inflammatory markers: IL-1 $\beta$, IL-4, IL-6, IL-10, TNF- $\alpha$, and CRP [27]. This tool has been developed over the past five years and it's used in different datasets across diverse populations in order to predict levels of inflammatory markers and related health outcomes [27]. Therefore, it is advised to be used when treating a patient's inflammatory status. The use of this tool is to classify the individual's diets, which demonstrates their immune system status with regards to inflammation, focusing on both anti-inflammatory and 
pro-inflammatory influence. Such status is observed as a score for each food item and component that were collected form each participant obtained from the 24-h dietary recall interviews (24HR) to positive or negative affect of inflammation levels. A score was given to each food and component dependent on the previous findings from reviewed journal articles (Figure 1 shows the previous studies were used with dietary inflammatory index). Accordingly, the scoring strategies of dietary inflammatory index on food parameter are one of three possible values, +1 if pro-inflammatory, 0 if produced no change in inflammatory marker, -1 if anti-inflammatory [27]. Specific compounds in the diet are believed to excrete anti-inflammatory actions, which include polyphenols [28].

DII has never been implemented on the Saudi population, which is considered an excellent potential tool to be used by physicians and clinical dietitians to assess inflammatory dietary parameters. The objectives of the current study are to assess inflammation in obese Saudi patients who are admitted for bariatric surgeries, to identify dietary factors related to inflammation and consider it in further recovery stages. This study is following Shivappa et. al. study [27] using a cross sectional study by $24 \mathrm{hr}$ recall and DII.

\section{Materials and Methods}

\subsection{Samples}

A total of 10 adults' volunteers, (aged between 18-50 years; female and male), recruited from king Abdul Aziz university hospital, Jeddah, Saudi Arabia. Fully informed and written consent was obtained in all cases and the study protocol was approved by Medicine College Ethics Committee at King AbdulAziz university (Reference NO413-15). Participants were admitted for a bariatric surgery in the year 2016. Inclusion criteria include a BMI above $\left(30 \mathrm{~kg} / \mathrm{m}^{2}\right)$ and free from chronic, inflammatory, and infectious diseases. Exclusion criteria included genetics obesity, patients on steroids based medications and on specific diet before the surgery. A descriptive analysis of volunteers has been carried (Table 1)

\subsection{4-hour recall}

The diet of the participants was assessed over a period of three days (2 weekdays and 1 weekend day) in which the respondent is asked to recall and describe all food and drinks consumed during the interview. Firstly, a quick list of any consumed foods is obtained; Secondary, record all information about the snacks and meals consumed including place and time; Thirdly, prompts for foods that may have been forgotten; Finally, the record and more details of portion sizes related to any consumed foods will be revised and completed [30]. The interviews were conducted in the surgical ward at king Abdulaziz University Hospital. The $24 \mathrm{~h}$ recall interviews were included in the questionnaires to assess nutritional, chemical and inflammatory status such as BMI, CRP, lipid profile, and signs of inflammation, medication, allergy).
Table 1. Descriptive analysis of volunteers

\begin{tabular}{|c|c|}
\hline Age (mean \pm SD) & $38.70 \pm 13.05$ \\
\hline \multicolumn{2}{|l|}{ Gender n (N\%) } \\
\hline Male & $3(30 \%)$ \\
\hline Female & $7(70 \%)$ \\
\hline Weight (kg) (mean \pm SD) & $107.65 \pm 12.68$ \\
\hline Hight (cm) (mean \pm SD) & $162 \pm 7.72$ \\
\hline BMI (kg/m2) (mean \pm SD) & $40.07 \pm 3.35$ \\
\hline \multicolumn{2}{|l|}{ BMI Obesity class n (N\%) } \\
\hline Obese class 1 & $2(20 \%)$ \\
\hline Obese class 2 & $2(20 \%)$ \\
\hline Obese class 3 & $6(60 \%)$ \\
\hline \multicolumn{2}{|c|}{ Do you have chronic diseases? n (N\%) } \\
\hline No & $6(60 \%)$ \\
\hline Yes & $4(40 \%)$ \\
\hline Diabetes & $2(20 \%)$ \\
\hline Hypertension & $1(10 \%)$ \\
\hline High cholesterol & $1(10 \%)$ \\
\hline CHD & $1(10 \%)$ \\
\hline \multicolumn{2}{|l|}{ Do you have allergies? n (N\%) } \\
\hline No & $9(90 \%)$ \\
\hline Yes & $1(10 \%)$ \\
\hline \multicolumn{2}{|c|}{ Do you have family disease history? n (N\%) } \\
\hline Diabetes & $7(70 \%)$ \\
\hline Hypertension & $2(20 \%)$ \\
\hline CHD & $1(10 \%)$ \\
\hline \multicolumn{2}{|c|}{ Do you take any of the following medication regularly? n (N\%) } \\
\hline No & $6(60 \%)$ \\
\hline Yes & $4(40 \%)$ \\
\hline \multicolumn{2}{|l|}{ Smoking n (N\%) } \\
\hline No & $8(80 \%)$ \\
\hline Yes & $2(20 \%)$ \\
\hline \multicolumn{2}{|c|}{ Did you receive breastfeeding? n (N\%) } \\
\hline No & $3(30 \%)$ \\
\hline Yes & $7(70 \%)$ \\
\hline
\end{tabular}

\subsection{Dietary Inflammatory Index}

The dietary intake of each participant were obtained from the 24-h recall interview and analyzed using Super Tracker website. Super tracker was used to collect all food and constituents used in inflammatory index except flavonoids intake. The flavonoids intake were collected using (phenol-explorer database website). After analysis of individual intake, the dietary inflammatory index was calculated by finding out the Z-score and percentile for each food firstly. The Z-score was calculated by subtracting the standard mean from the amount reported (the amount of food eaten by individual) and dividing the value by standard deviation for the food. (The standard mean and standard deviation are obtained from Figure 1). Then the Z-score converted to percentile to minimize the right skewing effect.( the symmetrical distribution will achieved with value centered on 0 (null) and the value between -1 (maximally anti-inflammatory) and +1 (maximally pro-inflammatory), each percentile score is doubled and then " 1 " is subtracted). After that, the centered percentile was multiplied by overall food parameter-specific inflammatory effect score (Figure 1) to identify the food parameter specific DII score. Finally, each food parameter-specific DII scores was summed to create the overall DII score for an individual which is range from -8 to $+8[27,31]$. 
Food parameters included in the dietary inflammatory index, inflammatory effect scores, and intake values from the global composite data set; Dietary Inflammatory Index Development Study, Columbia, SC, USA, 2011-2012

\begin{tabular}{|c|c|c|c|c|c|}
\hline Food par am eter & $\begin{array}{r}\text { Weighted number of } \\
\text { articles }\end{array}$ & $\begin{array}{c}\text { Raw inflammatory effect } \\
\text { score }\end{array}$ & $\begin{array}{r}\text { Overall inflamm atory } \\
\text { effect score }^{t}\end{array}$ & $\begin{array}{l}\text { Global daily m ean } \\
\text { intake }^{\ddagger} \text { (units/d) }\end{array}$ & $\mathrm{SD}^{\ddagger}$ \\
\hline Alcohol (g) & 417 & -0.278 & $-0 \cdot 278$ & 13.98 & 372 \\
\hline Vitamin $B_{12}(\mu \mathrm{g})$ & 122 & 0.205 & $0 \cdot 106$ & $5 \cdot 15$ & $2 \cdot 70$ \\
\hline Vitamin $\mathrm{B}_{6}(\mathrm{mg})$ & 227 & -0.379 & -0.365 & 1.47 & 0.74 \\
\hline$\beta$-Carotene $(\mu \mathrm{g})$ & 401 & -0.584 & -0.584 & 3718 & 1720 \\
\hline Caffeine (g) & 209 & $-0 \cdot 124$ & $-0 \cdot 110$ & 8.05 & 6.67 \\
\hline Carbohydrate (g) & 211 & $0 \cdot 109$ & 0.097 & $272 \cdot 2$ & $40 \cdot 0$ \\
\hline Cholesterol (mg) & 75 & 0.347 & $0 \cdot 110$ & $279 \cdot 4$ & $51 \cdot 2$ \\
\hline Energy (kcal) & 245 & $0 \cdot 180$ & $0 \cdot 180$ & 2056 & 338 \\
\hline Eugenol (mg) & 38 & -0.868 & $-0 \cdot 140$ & 0.01 & 0.08 \\
\hline Total fat (g) & 443 & 0.298 & 0.298 & $71 \cdot 4$ & $19 \cdot 4$ \\
\hline Fibre (g) & 261 & -0.663 & -0.663 & $18 \cdot 8$ & 49 \\
\hline Folic acid $(\mu g)$ & 217 & -0.207 & $-0 \cdot 190$ & $273 \cdot 0$ & $70 \cdot 7$ \\
\hline Garlic (g) & 277 & -0.412 & -0.412 & $4 \cdot 35$ & 290 \\
\hline Ginger (g) & 182 & -0.588 & -0.453 & $59 \cdot 0$ & $63 \cdot 2$ \\
\hline $\mathrm{Fe}(\mathrm{mg})$ & 619 & $0-032$ & 0.032 & $13 \cdot 35$ & 371 \\
\hline $\mathrm{Mg}(\mathrm{mg})$ & 351 & -0.484 & -0.484 & $310 \cdot 1$ & $139 \cdot 4$ \\
\hline MUFA (g) & 106 & -0.019 & -0.009 & $27 \cdot 0$ & $6 \cdot 1$ \\
\hline Niacin (mg) & 58 & $-1 \cdot 000$ & $-0 \cdot 246$ & $25 \cdot 90$ & $11 \cdot 77$ \\
\hline$n-3$ Fatty acids (g) & 2588 & -0.436 & -0.436 & 1.06 & 1.06 \\
\hline$n-6$ Fatty acids (g) & 924 & $-0 \cdot 159$ & $-0 \cdot 159$ & $10 \cdot 80$ & $7 \cdot 50$ \\
\hline Onion (g) & 145 & -0.490 & $-0 \cdot 301$ & $35 \cdot 9$ & $18 \cdot 4$ \\
\hline Protein (g) & 102 & 0.049 & 0.021 & $79 \cdot 4$ & $13 \cdot 9$ \\
\hline PUFA (g) & 4002 & -0.337 & -0.337 & $13 \cdot 88$ & 376 \\
\hline Riboflavin (mg) & 22 & -0.727 & -0.068 & 170 & 0.79 \\
\hline Saffron $(g)$ & 33 & $-1 \cdot 000$ & $-0 \cdot 140$ & 0.37 & $1 \cdot 78$ \\
\hline Saturated fat $(g)$ & 205 & 0.429 & 0.373 & $28 \cdot 6$ & $8 \cdot 0$ \\
\hline $\mathrm{Se}(\mu g)$ & 372 & $-0 \cdot 191$ & $-0 \cdot 191$ & $67 \cdot 0$ & $25 \cdot 1$ \\
\hline Thiamin (mg) & 65 & -0.354 & -0.098 & $1 \cdot 70$ & 0.66 \\
\hline Trans fat (g) & 125 & 0.432 & 0.229 & $3 \cdot 15$ & 375 \\
\hline Turmeric (mg) & 814 & -0.785 & -0.785 & $533 \cdot 6$ & $754 \cdot 3$ \\
\hline Vitamin $A(R E)$ & 663 & -0.401 & -0.401 & 983.9 & $518 \cdot 6$ \\
\hline Vitamin C (mg) & 733 & -0.424 & -0.424 & $118 \cdot 2$ & $43 \cdot 46$ \\
\hline Vitamin D $(\mu g)$ & 996 & -0.446 & -0.446 & $6 \cdot 26$ & $2 \cdot 21$ \\
\hline Vitamin $E(m g)$ & 1495 & -0.419 & -0.419 & $8 \cdot 73$ & 1.49 \\
\hline $\mathrm{Zn}(\mathrm{mg})$ & 1036 & $-0 \cdot 313$ & $-0 \cdot 313$ & $9 \cdot 84$ & $2 \cdot 19$ \\
\hline Green/black tea $(\mathrm{g})$ & 735 & -0.536 & -0.536 & $1 \cdot 69$ & 1.53 \\
\hline
\end{tabular}




\section{Results}

\subsection{4-hour recall}

Obese patients were assessed by 24-hour recall. In addition, the results showed that all the patients had proinflammatory score (Table 2).

Table 2. Descriptive analysis of DII level

\begin{tabular}{cccccc}
\hline & $\mathrm{N}$ & Minimum & Maximum & Mean & SE \\
\hline DII & 10.00 & 0.04 & 5.07 & 3.56 & 0.47 \\
\hline
\end{tabular}

\subsection{CRP \& lipid profile}

Blood tests have been carried to assess the CRP and lipid profile including LDL, HDL, TG and cholesterol. The results in (Table 3 ) show that mean level of CRP is higher than the normal range $(0-3 \mathrm{mg} / \mathrm{dl})$ among all patients.

Table 3. Descriptive analysis of biochemical data

\begin{tabular}{cccccc}
\hline & $\mathrm{N}$ & Minimum & Maximum & Mean & SE \\
\hline $\begin{array}{c}\text { C-reactive protein } \\
\text { (mg/dl) }\end{array}$ & 10 & 5.51 & 23.80 & 13.09 & 2.63 \\
LDL (mmol/l) & 10 & 3.50 & 5.64 & 4.24 & 0.70 \\
HDL (mmol/l) & 10 & 0.72 & 1.73 & 1.23 & 0.51 \\
TG (mmol/l) & 10 & 1.24 & 2.58 & 1.82 & 0.22 \\
Cholesterol & 10 & 3.28 & 7.69 & 5.05 & 0.62 \\
\hline
\end{tabular}

\subsection{CRP, Lipid Profile and Other Factors: Age, Gender, Allergy and Breastfeeding}

Some factors including age, gender, lipid profile, allergy and breast feeding are studied to examine the effect on CRP level. There was no significant correlation between age and the level of CRP however young adults had significantly higher level of CRP than older adults ( $P<0.05$, students T-test) (Table 4$)$. No association was seen between gender, lipid profile, allergy and breast feeding and CRP level (Table 5, Table 6, Table 7). Sample number in subject with allergy was too low to perform a statistical analysis to investigate whether allergy had an effect on CRP level.

Table 4. C-reactive protein level in young and older adults

\begin{tabular}{lcccc}
\hline & \multicolumn{3}{c}{$18-35$ young adults } & \multicolumn{2}{c}{$36-65$ older adults } \\
& Mean & SE & Mean & SE \\
\hline C-reactive protein $(\mathrm{mg} / \mathrm{dl})$ & 17.00 & 3.60 & $7.22^{*}$ & 0.53 \\
\hline
\end{tabular}

* denotes significant difference between age groups at $P<0.05$ significance level (student's T test)

Table 5. Lipid profile and c-reactive protein level in males and females

\begin{tabular}{l|c|c|c|c}
\hline \multirow{2}{*}{} & \multicolumn{4}{c}{ Gender } \\
\cline { 2 - 5 } & Mean & SE & Mean & SE \\
\hline C-reactive protein (mg/dl) & 11.35 & 5.33 & 13.83 & 3.23 \\
LDL (mmol/l) & 4.61 & 1.03 & 3.50 & $\cdot$ \\
HDL (mmol/l) &. &. & 1.23 & 0.51 \\
TG (mmol/l) & 2.15 & 0.32 & 1.49 & 0.15 \\
Cholesterol & 5.75 & 1.01 & 4.35 & 0.65 \\
\hline
\end{tabular}

There was no significant difference between gender groups (student's T test).
Table 6. C-reactive protein and allergy

\begin{tabular}{c|c|c|c|c}
\hline \multirow{2}{*}{} & \multicolumn{3}{|c|}{ do you have any type of allergy? } \\
\cline { 2 - 5 } & \multicolumn{2}{|c}{ No } & \multicolumn{2}{c}{ Yes } \\
\cline { 2 - 5 } & Mean & SE & Mean & SE \\
\hline C-reactive protein $(\mathrm{mg} / \mathrm{dl})$ & 12.10 & 2.72 & 22.00 & . \\
\hline
\end{tabular}

There was no significant difference between allergy groups (student's T test)

Table 7. C-reactive protein and breastfeeding

\begin{tabular}{c|c|c|c|c}
\hline \multirow{2}{*}{} & \multicolumn{3}{|c|}{ did you receive breastfeeding? } \\
\cline { 2 - 5 } & \multicolumn{2}{|c}{ No } & \multicolumn{2}{c}{ Yes } \\
\cline { 2 - 5 } & Mean & SE & Mean & SE \\
\hline C-reactive protein $(\mathrm{mg} / \mathrm{dl})$ & 17.14 & 5.31 & 11.35 & 3.01 \\
\hline
\end{tabular}

There was no significant difference between breastfed and non-breastfed groups (student's T test)

\subsection{Associations between diet and inflammation}

There was no difference in the DII or the intake of polyphenols and flavonoids between males and females (Table 8).

Table 8. Type and amount of polyphenols intake among female and male

\begin{tabular}{ccccc}
\hline & \multicolumn{2}{c}{ Male } & \multicolumn{2}{c}{ Female } \\
& Mean & SE & Mean & SE \\
\hline DII & 2.51 & 1.26 & 4.01 & 0.35 \\
flavan-3-ol & 7.94 & 3.97 & 12.31 & 9.97 \\
Flavones & 3.18 & 0.66 & 7.72 & 2.31 \\
Flavonols & 90.40 & 42.59 & 49.25 & 30.88 \\
Flavonones & 17.30 & 5.49 & 47.68 & 15.76 \\
Anthocyanidins & 13.23 & 6.90 & 0.95 & 0.95 \\
Isoflavones & 0.45 & 0.24 & 0.00 & 0.00 \\
\hline
\end{tabular}

DII was significantly positively correlated with the level of LDL $\left(\mathrm{P}<0.001, \mathrm{R}^{2}=0.969\right)$ and was negatively correlated with the level of HDL $\left(\mathrm{P}<0.001, \mathrm{R}^{2}=1\right)$. However, there was no association between the DII and TG, Cholesterol and CRP (Figure 2, Figure 3, Figure 4). There was no association between types and amount of polyphenols intake and level of CRP, except flavonols was strongly associated negatively with CRP $\left(\mathrm{p}<0.05, \mathrm{R}^{2}=0.425\right)$ (Figure 3$)$.

There was no significant difference between gender groups (student's T test)

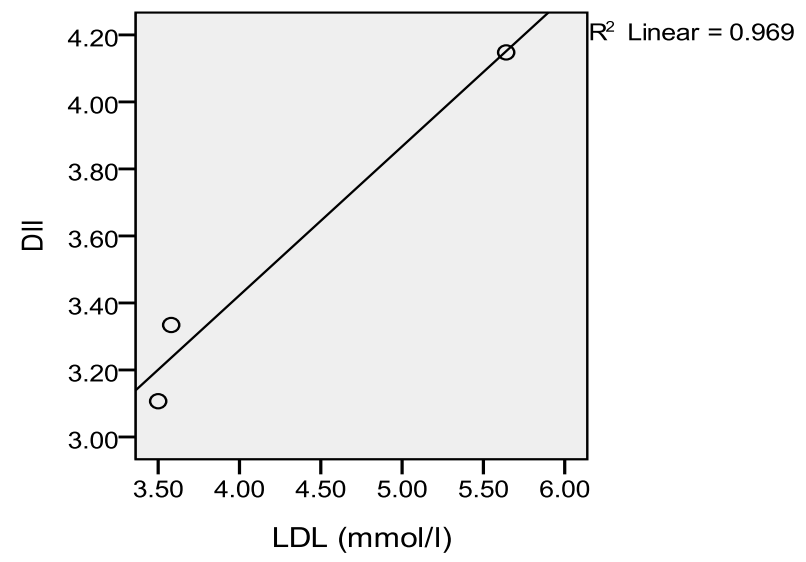

Figure 2. Relation between DII and LDL 


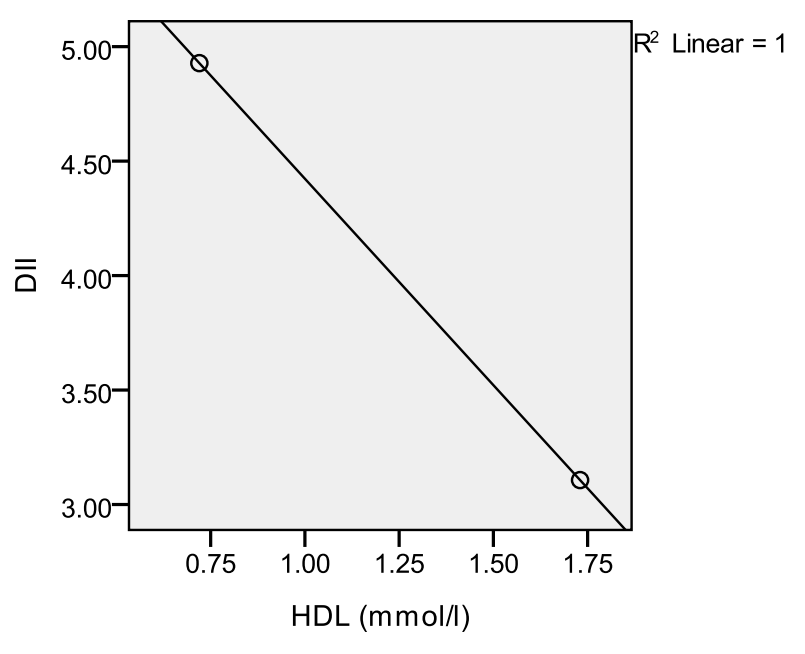

Figure 3. Relation between DII and HDL

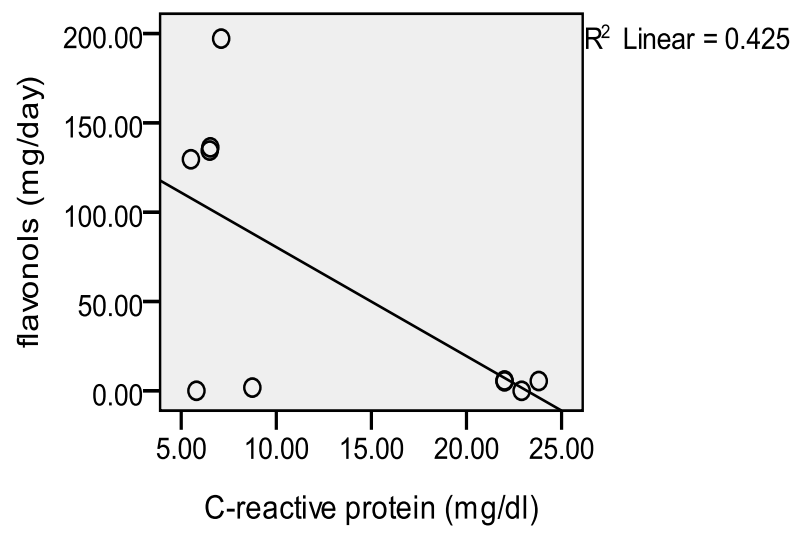

Figure 4. Relation between flavonols intake and C - reactive protein

\section{Discussion}

Epidemiological studies have proved that there is a strong association between inflammation and obesity, due to the action of macrophage [3]. In addition, diet has been associated with reducing inflammation, which include anti inflammatory agents, such as flavonoids, proved in vitro [23,32], and in vivo [33]. The aim of the present study is to examine the inflammatory status of obese patients. Initially the capacity of overall dietary patterns that promote inflammation in obese patients by using dietary inflammatory index tool. Our results showed that all the obese patients had an increased DII pro inflammatory score close to +1 . Our results were in agreement with canela et al study, where DII score was higher in people who consume less Mediterranean diet, fruits and vegetables that are rich in polyphenols [34]. As it showed in our results all subjects had a pro inflammatory, which may be due to consuming very little amounts of polyphenols which affect their DII score to be close to +1 . This study has also tested the association between DII scores and BMI for the first time in Saudi Arabia, where it showed a positive association. People with BMI > 29.5 $\mathrm{kg} / \mathrm{m}^{2}$ are always at a state of chronic low grade of inflammation [3]. DII is a new instrument used for assessing the inflammatory potential of the diet [35]. Therefore, it does need further investigation to explain the relationship between DII and other factors such as BMI. Dietary inflammatory index (pro inflammatory score close to +1) and anthropometric measures of obesity(BMI > $29.5 \mathrm{~kg} / \mathrm{m}^{2}$ ) study is the only study that was found to show the direct association between the DII and obesity, and supports the hypothesis that diet low in fruits and vegetables, legumes and nuts may have a role in the development of obesity through inflammatory modulation mechanisms [34] one of the previous mentioned limitation is that unclear whether obese individuals are more likely to choose pro inflammatory diets, or if pro inflammatory diets contributes to promoting obesity. However, diet with an anti inflammatory potential can play an important role in correcting obese inflammatory status and help to prevent consequences associated with chronic inflammation [34]. According to several studies, obesity is strongly associated with a state of chronic low-grade of inflammation [5]. We have observed that there is an increased level of CRP among all subjects, which was also seen in Finucane study, where it showed that obese have an increased level of circulating inflammatory markers such as C-reactive protein and other pro inflammatory cytokines such as TNF, IL-6 and IL-1B produced by M1 [3]. Another studies support the result and showed that BMI has a significant positive association with CRP level $[4,5,33,35]$. All this refers to the mechanism of inflammation associated with obesity as the progressive immune cells filtration occurs into obese adipose tissue macrophages which emerge into their body leading to a state of chronic low grade of inflammation [3]. The effect of age on serum C-reactive protein is debated, some studies shows no association [36] and while others believe, it increases with age. This study confirms the findings of Miller et al. that there was no significant correlation between age and the level of CRP, according to a population-based study, there was a statistically significant unadjusted positive correlation (P 0.0001) between CRP and age. Our result shows no association was seen between gender and CRP level due to low number of participants recruited in this study. In contrast, large contemporary, U.S. population-based sample study, they observed that the median CRP level was almost twice as high in women compared with men. However, their study was similar to our finding that CRP levels were identical in women and men. Another factor is breast feeding and there is a conflict whether breast feeding has a protective role on inflammation or not. According to questionnaires customized on inflammation, results have shown no association was seen between breast-feeding and CRP level. The previous studies have been shown there was no relation between infant breastfeeding and CRP levels in men [37]. In contrast, there was a strong relation between duration of breast-feeding for three or more months and lower CRP levels in women. Although there some studies have reported that no significant increases in serum CRP between breastfed participants and those never breastfed [38]. There are several possible explanations, where breastfeeding might influence low-grade inflammation only in people who are predisposed to genetic problem. Also, breastfeeding might affect inflammatory markers only in subgroups of the population who have more pronounced low-grade inflammatory status. Another hypothesis is that infancy is too early to detect a significant benefit of breastfeeding on 
inflammation. Our suggest that need more studies, also need more duration because a shorter duration of breastfeeding, as well as never breastfeeding, may affects the association with the inflammation .On the other hand, gender may be taken into consideration, because it was associated with lower food intakes by women. Also in the present study most of the women were eating less than recommended dietary intake RDI and interview bias could be the reason behind such low intake in the obese subjects. This fact influences polyphenol intake as it was shown in [39] study were the average polyohenol intake in women was 817 mglday and 919 mglday in men which could contribute to a change in the dietary inflammatory index score. In another study polyphenol intakes was higher in the men (1172 mg/day) compared to the women (1031 $\mathrm{mg} /$ day) and plant food categories such as beverages, vegetables, fruits and cereals were found to be significant sources of polyphenols [40]. In contrast a study conducted in the US showed higher intake of Pro-anthocyanidins a polymers of flavan-3-ols in women $(\mathrm{P}<0.01)$ and the major food sources of total PA intake in the U.S. diet were tea and legumes followed by wine [41]. Our findings showed no significant differences in the intake of polyphenols in both genders (Table 7). This indicates that polyphenols intake may vary due to many reasons, which include different dietary habits among countries, for example western diet has low amount of polyphenols compared to Mediterranean diet [42] due to high amount of red meat in the western diet which are likely to cause detrimental effects on polyphenol bio-accessibility [43], compare to Mediterranean diet which is rich in polyphenol compounds such as virgin olive oil (oleuropein and hydroxytyrosol) and red wine (resveratrol and quercetin) [44]. In our study, the total energy intake of obese subjects was lower than the recommended dietary intake (mean=1272 kcal/day), but low polyphenol intake by the individuals contributed to higher DII score (more pro-inflammatory) (Table 2). With regards to the relation between DII and gender [45] showed higher score in females than males, this was partially due to the fact that males had higher absolute intake amounts of many anti-inflammatory components of the DII this supported the results in the present study in which females had slightly higher DII score than male, but those results did not achieve statistical significance. In contrast with our finding a study showed controversial results in which the DII score was higher in male [46]. The differences among gender were due to underreporting, in which men gave more details about there diet and more accurate portion size than women during the interview. Lipid profile and weight status can be influenced by diet [47]. It was observed from the present study that DII was significantly positively correlated with the level of LDL $(\mathrm{P}<0.001$, $\mathrm{R}^{2}=0.969$ ) (Figure 1). Similarity, a significant correlation was detected by shivappa et al, where a significant increase in LDL level associated with DII level [48]. High DII contribute to a pro-inflammatory status (DII reference) and LDL can yield oxidize phospholipids that induce inflammatory response [49]. Although, However, with regards to HDL, (Figure 2) showed that DII was negatively correlated with the level of HDL $(\mathrm{P}<0.001$, $\mathrm{R}^{2}=1$ ). In contrast, with et al, show that there is no statistically difference between DII and HDL [50]. On the other hand, shivappa et al, have shown a normal level of HDL associated with DII [48]. This result may be due to we only have two patient they have HDL level, so our result maybe not representative for the population. In the future, we should assess the HDL level for all patient to get significant result.

The relation between polyphenols intake and CRP level is interesting, because both are associated with inflammation [51]. Chun et al, show that total flavonoid, individual flavonols, anthocyanidin, and isoflavone intake were significantly inversely associated with CRP [51]. Our study show in (Figure 3) that there was no association between types and amount of polyphenols intake and level of CRP, except flavonols was strongly associated negatively with CRP $\left(p<0.05, R^{2}=0.425\right)$. The mechanism behind this result is still unclear according to several studies $[52,53,54,55,56]$. However, in the present study this result may be due to small sample size, and the DII only estimate the intake of 6 type of poly phenols which include (flavan-3-ol, flavones, flavonols, flavonones, anthocyanidins, isoflavones). Although the Inflammatory Index assesses diet as a whole, it was created using articles that examined the effect of single nutrients and other dietary constituents on inflammation. Linking nutrients to obesity outcomes can yield weaker relationships compared with food or dietary pattern intake [57]. Another possible limitation of the Inflammatory Index is publication bias were Inflammatory Index is dependent on the published literature. The actual number of participants recruited in this study was half of the calculated sample size due to limited bariatric surgeries in the research period. Therefore, in order to optimize dietary inflammatory index alone in the future among Saudi population we need bigger sample size. Another limitation was in the 24-hour recall in which all the interviews were conducted after the surgery because most of the patients did not administer one day prior to the surgery so that leaded to a bias in the interview. This is the first study to date in Saudi Arabia to apply the DII test and link it with C-reactive protein to asses inflammatory status of obese subjects and link to their diet. In conclusion, DII can be used in the future as a new tool in the routine assessment of the inflammatory potential for the diet which can be applied to obese population in Saudi Arabia. In the next step of this research we will study the therapeutic application of one of the polyphenols reviewed in literature to induce an anti-inflammatory response in obesity.

\section{Acknowledgements}

We would like to express our sincere gratitude to the staff and patients admitted in the surgery ward for their support of the project.

\section{References}

[1] Nguyen DM, El-Serag HB. The Epidemiology of Obesity. Gastroenterology Clinics. 39(1): 1-7.

[2] Memish ZA, El Bcheraoui C, Tuffaha M, Robinson M, Daoud F, Jaber S. Obesity and Associated Factors - Kingdom of Saudi Arabia, 2013. Preventing Chronic Disease. 2014; 11: E174. 
[3] Finucane OM, Reynolds CM, McGillicuddy FC, Roche HM. Insights into the role of macrophage migration inhibitory factor in obesity and insulin resistance. Proc Nutr Soc. 2012; 71(4): 622-33.

[4] Bullo M, Casas-Agustench P, Amigo-Correig P, Aranceta J, SalasSalvado J. Inflammation, obesity and comorbidities: the role of diet. Public health nutrition. 2007; 10(10a): 1164-72.

[5] Emanuela F, Grazia M, Marco de R, Maria Paola L, Giorgio F, Marco B. Inflammation as a Link between Obesity and Metabolic Syndrome. Journal of nutrition and metabolism. 2012; 2012: 476380.

[6] center Wm. obesity and overweight 2015 [Available from: http://www.who.int/mediacentre/factsheets/fs311/en/.

[7] Pi-Sunyer FX. Obesity: criteria and classification. Proc Nutr Soc. 2000; 59(4): 505- 9.

[8] Calder PC, Ahluwalia N, Brouns F, Buetler T, Clement K, Cunningham K, et al. Dietary factors and low-grade inflammation in relation to overweight and obesity. British Journal of Nutrition. 2011; 106(SupplementS3): S1-S78.

[9] Romeo GR, Lee J, Shoelson SE. Metabolic Syndrome, Insulin Resistance, and Roles of Inflammation - Mechanisms and Therapeutic Targets. Arteriosclerosis, thrombosis, and vascular biology. 2012; 32(8): 1771-6.

[10] Lumeng CN, Bodzin JL, Saltiel AR. Obesity induces a phenotypic switch in adipose tissue macrophage polarization. J Clin Invest. 2007; 117(1): 175-84.

[11] Bastard JP, Maachi M, Lagathu C, Kim MJ, Caron M, Vidal H, et al. Recent advances in the relationship between obesity, inflammation, and insulin resistance. European cytokine network. 2006; 17(1): 4-12.

[12] Wood IS, de Heredia FP, Wang B, Trayhurn P. Cellular hypoxia and adipose tissue dysfunction in obesity. Proceedings of the Nutrition Society. 2009; 68(04): 370-7.

[13] Greenberg AS, Obin MS. Obesity and the role of adipose tissue in inflammation and metabolism. The American journal of clinical nutrition. 2006; 83(2): 461s-5s.

[14] Shoelson SE, Lee J, Goldfine AB. Inflammation and insulin resistance. Journal of Clinical Investigation. 2006; 116(7): 1793-801.

[15] Cinti S, Mitchell G, Barbatelli G, Murano I, Ceresi E, Faloia E, et al. Adipocyte death defines macrophage localization and function in adipose tissue of obese mice and humans. J Lipid Res. 2005; 46(11): 2347-55.

[16] Coenen KR, Gruen ML, Chait A, Hasty AH. Diet-induced increases in adiposity, but not plasma lipids, promote macrophage infiltration into white adipose tissue. Diabetes. 2007; 56(3): 564-73.

[17] Strissel KJ, Stancheva Z, Miyoshi H, Perfield JW, 2nd, DeFuria J, Jick Z, et al. Adipocyte death, adipose tissue remodeling, and obesity complications. Diabetes. 2007; 56(12): 2910-8.

[18] Aron-Wisnewsky J, Tordjman J, Poitou C, Darakhshan F, Hugol D, Basdevant A, et al. Human Adipose Tissue Macrophages: M1 and M2 Cell Surface Markers in Subcutaneous and Omental Depots and after Weight Loss. The Journal of Clinical Endocrinology \& Metabolism. 2009; 94(11): 4619-23.

[19] Fujisaka S, Usui I, Bukhari A, Ikutani M, Oya T, Kanatani Y, et al. Regulatory mechanisms for adipose tissue M1 and M2 macrophages in diet-induced obese mice. Diabetes. 2009; 58(11): 2574-82.

[20] Satoh N, Shimatsu A, Himeno A, Sasaki Y, Yamakage H, Yamada $\mathrm{K}$, et al. Unbalanced M1/M2 phenotype of peripheral blood monocytes in obese diabetic patients: effect of pioglitazone. Diabetes Care. 2010; 33(1): e7.

[21] Surmi BK, Hasty AH. Macrophage infiltration into adipose tissue: initiation, propagation and remodeling. Future Lipidol. 2008; 3(5) 545-56.

[22] Ando C, Takahashi N, Hirai S, Nishimura K, Lin S, Uemura T, et al. Luteolin, a food-derived flavonoid, suppresses adipocytedependent activation of macrophages by inhibiting JNK activation. FEBS Letters. 2009; 583(22): 3649-54.

[23] Kang JH, Kim CS, Han IS, Kawada T, Yu R. Capsaicin, a spicy component of hot peppers, modulates adipokine gene expression and protein release from obese-mouse adipose tissues and isolated adipocytes, and suppresses the inflammatory responses of adipose tissue macrophages. FEBS Lett. 2007; 581(23): 4389-96.

[24] Shirakawa J, Fujii H, Ohnuma K, Sato K, Ito Y, Kaji M, et al. Diet-Induced Adipose Tissue Inflammation and Liver Steatosis
Are Prevented by DPP-4 Inhibition in Diabetic Mice. Diabetes. 2011; 60(4): 1246-57.

[25] Overman A, Bumrungpert A, Kennedy A, Martinez K, Chuang CC, West $T$, et al. Polyphenol-rich grape powder extract (GPE) attenuates inflammation in human macrophages and in human adipocytes exposed to macrophage-conditioned media. Int J Obes. 2010; 34(5): 800-8.

[26] Min SY, Yang H, Seo SG, Shin SH, Chung MY, Kim J, et al. Cocoa polyphenols suppress adipogenesis in vitro and obesity in vivo by targeting insulin receptor. Int J Obes (Lond). 2013; 37(4): 584-92.

[27] Shivappa N, Steck SE, Hurley TG, Hussey JR, Hébert JR. Designing and developing a literature-derived, population-based dietary inflammatory index. Public health nutrition. 2014; 17(8): 1689-96.

[28] Del Rio D, Rodriguez-Mateos A, Spencer JPE, Tognolini M, Borges G, Crozier A Dietary (Poly)phenolics in Human Health: Structures, Bioavailability, and Evidence of Protective Effects Against Chronic Diseases. Antioxid Redox Signal. 2013; 18(14): 1818- 92.

[29] Ding Q, Mracek T, Gonzalez-Muniesa P, Kos K, Wilding J, Trayhurn $\mathrm{P}$, et al. Identification of macrophage inhibitory cytokine- 1 in adipose tissue and its secretion as an adipokine by human adipocytes. Endocrinology. 2009; 150(4): 1688-96.

[30] Wrieden W, Peace H, Armstrong J, Barton K, editors. A short review of dietary assessment methods used in National and Scottish Research Studies. Briefing Paper Prepared for: Working Group on Monitoring Scottish Dietary Targets Workshop Edinburgh; 2003.

[31] Cavicchia PP, Steck SE, Hurley TG, Hussey JR, Ma Y, Ockene IS et al. A New Dietary Inflammatory Index Predicts Interval Changes in Serum High-Sensitivity CReactive Protein. The Journal of Nutrition. 2009; 139(12): 2365-72.

[32] Buttari B, Profumo E, Segoni L, \#x, Arcangelo D, Rossi S, et al. Resveratrol Counteracts Inflammation in Human M1 and M2 Macrophages upon Challenge with 7-Oxo-Cholesterol: Potential Therapeutic Implications in Atherosclerosis. Oxidative Medicine and Cellular Longevity. 2014; 2014: 12.

[33] de Jesus Soares T, Volpini RA, Francescato HD, Costa RS, da Silva CG, Coimbra TM. Effects of resveratrol on glycerol-induced renal injury. Life Sci. 2007; 81(8): 647-56.

[34] Ruiz-Canela M, Zazpe I, Shivappa N, Hebert JR, Sanchez-Tainta A, Corella D, et al. Dietary inflammatory index and anthropometric measures of obesity in a population sample at high cardiovascular risk from the PREDIMED (PREvencion con DIeta MEDiterranea) trial. The British journal of nutrition. 2015; 113(6): 984-95.

[35] Forsythe LK, Wallace JM, Livingstone MB. Obesity and inflammation: the effects of weight loss. Nutrition research reviews. 2008; 21(2): 117-33.

[36] Feldman M SS. Is CRP, like ESR, Age and Gender Dependent?. Rheumatology (Sunnyvale). 2014; 4(2): 1-4.

[37] Williams MJA, Williams SM, Poulton R. Breast feeding is related to $\mathrm{C}$ reactive protein concentration in adult women. Journal of Epidemiology and Community Health. 2006; 60(2): 146-8.

[38] Roszkowska R, Taranta-Janusz K, Tenderenda-Banasiuk E, Wasilewska A. The effects of breastfeeding on serum asymmetric dimethylarginine levels and body composition in children. Breastfeeding medicine: the official journal of the Academy of Breastfeeding Medicine. 2015; 10(1): 38-44.

[39] Ovaskainen ML, Torronen R, Koponen JM, Sinkko H, Hellstrom J, Reinivuo $\mathrm{H}$, et al. Dietary intake and major food sources of polyphenols in Finnish adults. The Journal of Nutrition. 2008; 138(3): 562-6.

[40] Zujko ME, Witkowska AM, Waskiewicz A, Sygnowska E. Estimation of dietaryvintake and patterns of polyphenol consumption in Polish adult population. Advances in medical sciences. 2012; 57(2): 375-84.

[41] Wang Y, Chung SJ, Song WO, Chun OK. Estimation of daily proanthocyanidin intake and major food sources in the U.S. diet. The Journal of Nutrition. 2011; 141(3): 447-52.

[42] Ogce F, Ceber E, Ekti R, Oran NT. Comparison of mediterranean, Western and Japanese diets and some recommendations. Asian Pacific journal of cancer prevention: APJCP. 2008; 9(2): 351-6.

[43] Bohn T. Dietary factors affecting polyphenol bioavailability. Nutrition reviews. 2014; 72(7): 429-52. 
[44] Scoditti E, Calabriso N, Massaro M, Pellegrino M, Storelli C, Martines G, et al. Mediterranean diet polyphenols reduce inflammatory angiogenesis through MMP-9 and COX-2 inhibition in human vascular endothelial cells: A potentially protective mechanism in atherosclerotic vascular disease and cancer. Archives of Biochemistry and Biophysics. 2012; 527(2): 81-9.

[45] Wirth MD, Shivappa N, Steck SE, Hurley TG, Hebert JR. The dietary inflammatory index is associated with colorectal cancer in the National Institutes of Health-American Association of Retired Persons Diet and Health Study. The British journal of nutrition. 2015; 113(11): 1819-27.

[46] Wirth MD, Hébert JR, Shivappa N, Hand GA, Hurley TG, Drenowatz C, et al. Anti-inflammatory Dietary Inflammatory Index scores are associated with healthier scores on other dietary indices. Nutrition Research. 36(3): 214-9.

[47] Callahan E. Changes in weight loss and lipid profiles after a dietary purification program: a prospective case series. Journal of Chiropractic Medicine. 2013; 12(1): 30-8.

[48] Shivappa N, Steck SE, Hurley TG, Hussey JR, Ma Y, Ockene IS, et al. A population-based dietary inflammatory index predicts levels of C-reactive protein in the Seasonal Variation of Blood Cholesterol Study (SEASONS). Public health nutrition. 2014; 17(8): 1825-33.

[49] Navab M, Berliner JA, Subbanagounder G, Hama S, Lusis AJ, Castellani LW, et al. HDL and the inflammatory response induced by LDL-derived oxidized phospholipids. Arteriosclerosis, thrombosis, and vascular biology. 2001; 21(4): 481-8.

[50] Wirth MD, Burch J, Shivappa N, Violanti JM, Burchfiel CM, Fekedulegn D, et al. Association of a dietary inflammatory index with inflammatory indices and metabolic syndrome among police officers. Journal of occupational and environmental medicine / American College of Occupational and Environmental Medicine. 2014; 56(9): 986-9.

[51] Chun OK, Chung S-J, Claycombe KJ, Song WO. Serum CReactive Protein Concentrations Are Inversely Associated with Dietary Flavonoid Intake in U.S. Adults. The Journal of Nutrition. 2008; 138(4): 753-60.

[52] De Bacquer D, Clays E, Delanghe J, De Backer G. Epidemiological evidence for an association between habitual tea consumption and markers of chronic inflammation. Atherosclerosis. 2006; 189(2): 428-35.

[53] Gao X, Bermudez OI, Tucker KL. Plasma C-reactive protein and homocysteine concentrations are related to frequent fruit and vegetable intake in Hispanic and non- Hispanic white elders. The Journal of Nutrition. 2004; 134(4): 913-8.

[54] Widlansky ME, Duffy SJ, Hamburg NM, Gokce N, Warden BA, Wiseman S, et al. Effects of black tea consumption on plasma catechins and markers of oxidative stress and inflammation in patients with coronary artery disease. Free radical biology \& medicine. 2005; 38(4): 499-506.

[55] Esmaillzadeh A, Kimiagar M, Mehrabi Y, Azadbakht L, Hu FB, Willett WC. Fruit and vegetable intakes, C-reactive protein, and the metabolic syndrome. The American journal of clinical nutrition. 2006; 84(6): 1489-97.

[56] Steptoe A, Gibson EL, Vuononvirta R, Hamer M, Wardle J, Rycroft JA, et al. The effects of chronic tea intake on platelet activation and inflammation: a double-blind placebo controlled trial. Atherosclerosis. 2007; 193(2): 277-82.

[57] Hu FB. Dietary pattern analysis: a new direction in nutritional epidemiology. Current opinion in lipidology. 2002; 13(1): 3-9. 